Open Access

\title{
HDAC3 role in medication consumption in medication overuse headache patients: a pilot study
}

Claudia Pisanu', Stefano Caproni ${ }^{2}$, Donatella Congiu', Letizia M. Cupini ${ }^{3}$, Alessio Squassina ${ }^{1}$, George P. Patrinos ${ }^{4^{*}}$, Ilenia Corbelli ${ }^{2}$, Paolo Calabresi ${ }^{2,5}$, Maria Del Zompo ${ }^{1,6^{*}}$ and Paola Sarchielli ${ }^{2}$

\begin{abstract}
Background: Medication overuse headache $(\mathrm{MOH})$ is a common and debilitating disorder characterized by generation, perpetuation, and persistence of intense chronic migraine, caused by overuse of analgesics, triptans, or other acute headache compounds. It has been suggested that $\mathrm{MOH}$ could share some pathogenetic mechanisms with other kinds of drug addiction. In this regard, histone deacetylases 3 (HDAC3) seems to have a role in the memory processes involved in extinction of drug-seeking behavior in animal models. HDAC3 is inhibited by sodium valproate, a drug with proven efficacy in $\mathrm{MOH}$. Recent evidence suggests an involvement of genetic factors in predisposition to medication overuse.

Results: In this association study, we sequenced all exons, intron/exon junctions, and 3'-5'UTR regions of HDAC3 in $23 \mathrm{MOH}$ patients to investigate its role in medication overuse. Associations between genotypes with continuous and dichotomous clinical characteristics were tested by multivariate analysis and Fisher's exact test, respectively. Sequencing of HDAC3 revealed six single-nucleotide polymorphisms. The G allele of rs 2530223 was significantly associated with the number of acute medications/month used and with the number of days/month in which medications were used ( $p=0.006$ and $p=0.007$, respectively), but neither with headache frequency or intensity. None of the single-nucleotide polymorphisms (SNPs) was associated with clinical characteristics or response to sodium valproate.
\end{abstract}

Conclusions: $\mathrm{HDAC} 3$ could be implicated in excessive medication consumption in $\mathrm{MOH}$ patients. Our preliminary findings provide support for the need of further investigation on larger independent samples to confirm and extend the role of HDAC3 in medication overuse headache.

Keywords: Medication overuse headache, HDAC3, Sequencing, Sodium valproate

\section{Background}

Medication overuse headache $(\mathrm{MOH})$ is a common and debilitating disorder characterized by generation, perpetuation, and persistence of intense chronic migraine, caused by overuse of analgesics, triptans, or other acute headache compounds [1]. Epidemiological studies show that $\mathrm{MOH}$ has become the third most frequent type of

\footnotetext{
* Correspondence: gpatrinos@upatras.gr; delzompo@unica.it

${ }^{4}$ Department of Pharmacy, University of Patras, School of Health Sciences, Patras, Greece

${ }^{1}$ Section of Neurosciences and Clinical Pharmacology, Department of Biomedical Sciences, University of Cagliari, SP8 Km. 0,700, 09042 Monserrato (CA), Italy

Full list of author information is available at the end of the article
}

headache, with a prevalence of $1-2 \%$ of the general population [2].

An increased risk of developing $\mathrm{MOH}$ has been shown in subjects with a family history of medication, drug, or alcohol abuse, suggesting a genetic predisposition to medication overuse [3]. Moreover, genetic variants have been suggested to have a role in migraine transformation into $\mathrm{MOH}$ [4], success of detoxification therapy [5], and relapse during the first year of follow-up [6].

The use of acute medication with psychotropic effects (barbiturates and opioids) has been associated with increased risk of migraine chronification [7]. $\mathrm{MOH}$ 
patients show symptoms of withdrawal [3], a high rate of relapses [8], and decision-making deficits [9].

A neuroimaging study with $18 \mathrm{~F}-$-fluorodeoxyglucose positron-emission tomography in $\mathrm{MOH}$ patients showed that a number of dysmetabolic brain areas involved in pain-processing recovered after withdrawal of medication, while long-lasting dysfunction was observed in orbitofrontal cortex [10], an area suggested to be involved in drug dependence and addiction [11]. A recent study has also identified persistent dysfunctions in the mesocorticolimbic dopamine circuit in $\mathrm{MOH}$ patients [12]. On the basis of this evidence, it has been speculated that $\mathrm{MOH}$ shares some pathogenetic mechanisms with other kinds of drug addiction [13, 14].

Histone deacetylases 3 (HDAC3) is a protein expressed in almost all tissues, including brain [15], responsible for the deacetylation of lysine residues of the core histones. Among other functions, HDAC3 has been shown to be involved in negative regulation of long-term memory formation [16]. It has been suggested that molecular mechanisms underlying drug overuse are similar to those regulating long-term associative memory [17]. HDAC3 inhibition has been found to increase the memory processes involved in the extinction of drug-seeking behavior in animal models [18]. Moreover, HDAC3 expression has recently been found to be increased in rat dorsal striatal neurons which were activated during drug-seeking tests after prolonged methamphetamine withdrawal compared to nonactivated Fos-negative neurons [19].

Interestingly, this enzyme is inhibited by sodium valproate (VPA), an anticonvulsant and mood stabilizer approved by FDA for migraine prevention and shown to be effective in the treatment of chronic daily headache $[20,21]$. Although VPA has been proven to be efficacious in the treatment of $\mathrm{MOH}$ with a history of migraine without aura, the exact mechanism of action of this drug in $\mathrm{MOH}$ is not known, and a large percentage of treated patients do not respond [22].
In this study, we carried out an explorative investigation on the role of $H D A C 3$ genetic variants in medication overuse and response to VPA treatment in a sample of patients with $\mathrm{MOH}$ by sequencing of the functional regions of the gene.

\section{Results}

Sequencing of the $H D A C 3$ gene revealed five intronic single-nucleotide polymorphisms (SNP) (rs1421896, rs32954, rs2547547, rs149330805, and rs41290601) and the synonym variant located in exon 3, rs2530223 (Fig. 1). Percentages of successfully genotyped samples were $90 \%$ for rs1421896 and rs32954, and $100 \%$ for all the others SNPs. Genotyping was successful in all individuals. Rs32954 had a minor allele frequency (MAF) lower than 0.01 and was excluded from the analyses. All SNPs were already present in public databases (UCSC Genome Bioinformatics; NCBI), and no new variants were identified in our patients. All SNPs were in Hardy-Weinberg equilibrium (HWE) in our sample.

Multivariate analysis showed that rs2530223 was significantly associated with the number of acute medications/ month used and days/month in which acute medications were used ( $p=0.02$ and eta squared $=0.32$ for both variables), but not with frequency or intensity of headache. Specifically, GA and GG carriers showed a higher medication abuse compared to AA carriers. Since the means of the associated variables were similar for GA and GG carriers, we hypothesized a recessive model for the effect of the A allele on medication consumption, and we used this model for subsequent analysis. When we grouped $\mathrm{G}$ allele carriers, we found higher significance for number of drugs/month abused and days/month of drug abuse for both variables $(p=0.006$, eta squared $=0.31 ; p=0.007$, eta squared $=0.31$, respectively; Table 1$)$. G carriers were not different from other subjects in regard to age or $\mathrm{MOH}$ duration (Table 1). Although not significant, there was a sex difference in our groups (in $G$ carriers female

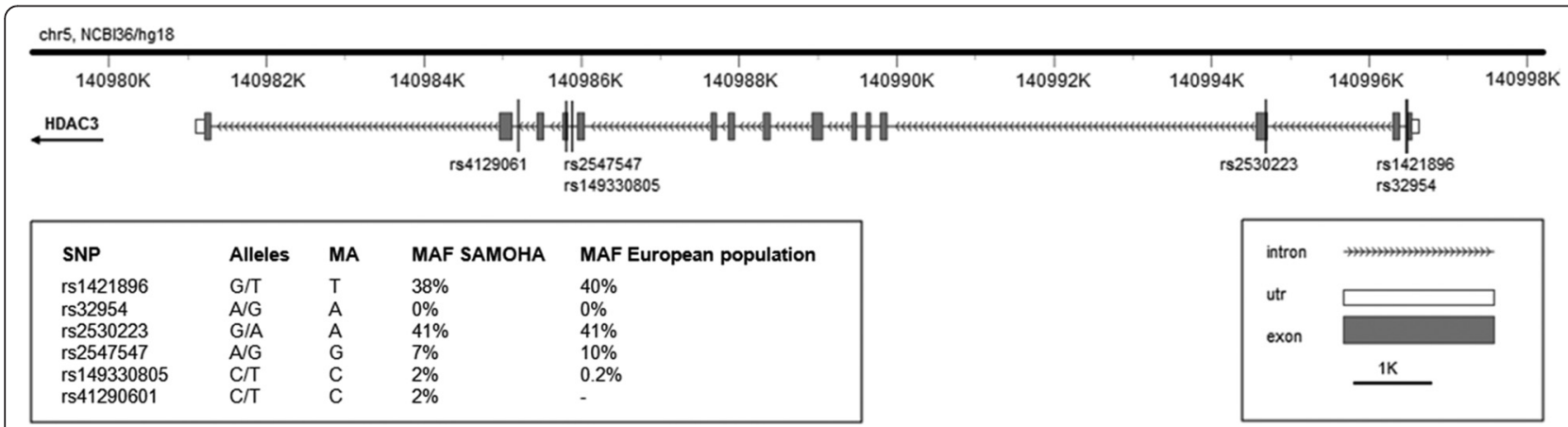

Fig. 1 Gene diagram of HDAC3 gene including SNPs identified in our sample. Abbreviations: SNP single-nucleotide polymorphism, MA minor allele, MAF minor allele frequency. Allele frequencies for European population are reported accordingly to 1000 Genome Project [29]; data obtained through VarioWatch [23]. The diagram was created with FancyGene [31] 
Table 1 Association between clinical characteristics and genotypes of rs2530223

\begin{tabular}{|c|c|c|c|c|c|}
\hline & $\mathrm{AA}$ & GA/GG & Statistics & $p$ value & Eta squared \\
\hline Women/men & $5 / 0$ & $12 / 6$ & & 0.27 & \\
\hline Age (years) & $42.2 \pm 10.9$ & $43.1 \pm 9.5$ & 0.18 & 0.5 & \\
\hline $\mathrm{MOH}$ duration & $3.9 \pm 3.8$ & $5.4 \pm 3.6$ & 0.81 & 0.37 & \\
\hline Number of headache days/month & $18.6 \pm 1.8$ & $21.9 \pm 4.1$ & 3.04 & 0.09 & 0.12 \\
\hline Headache intensity & $2.0 \pm 0.7$ & $2.2 \pm 0.6$ & 0.44 & 0.51 & 0.02 \\
\hline Number of drugs/month abused & $14.2 \pm 4.1$ & $23.8 \pm 6.7$ & 9.47 & 0.006 & 0.30 \\
\hline Days/month of drug abuse & $11.4 \pm 2.6$ & $19.0 \pm 5.3$ & 9.06 & 0.007 & 0.31 \\
\hline VPA responders/nonresponders & $4 / 1$ & $9 / 9$ & - & 0.33 & \\
\hline
\end{tabular}

Continuous data are expressed in mean \pm standard deviation; dichotomous data are expressed as frequencies

Headache intensity was measured with a 4-point scale with $0=$ no pain, $1=$ mild headache, $2=$ moderate headache, $3=$ severe headache. Headache attacks and drugs used during the baseline period were collected using a headache diary. Additional information on how these variables were collected is available in Sarchielli et al., 2014 [22]. Significant variables are indicated in bold

$\mathrm{MOH}$ medication overuse headache, VPA sodium valproate

prevalence was $66 \%$, while it was $100 \%$ among G noncarriers). For the numbers of drug/month abused, sex did not give a significant contribution to the model when tested as a covariate $(p=0.7)$. For the number of days/ month in which medications were used, sex was significant as a covariate $(p=0.042)$, and the model including sex and rs2530223 was significantly associated with numbers of days/month of abuse (model: $p=0.003$; rs2530223: $p=0.022$; sex: $p=0.042$ ). When correcting for headache frequency, the impact of rs 2530223 on number of medications used and days of abuse remained significant ( $p=0.03$ for both variables).

No HDAC3 SNP was associated with response to VPA in our sample (data not shown). If used as a covariate in the linear model including rs2530223 and numbers of days/month of abuse, VPA response gave a nonsignificant contribution $(p=0.8)$, while the contribution of the SNP was still significant $(p=0.01)$. Thus, a different response to VPA does not seem to be influencing the hypothesized association between rs2530223 and medication consumption.

The analysis conducted with VarioWatch [23] showed that rs2530223 was predicted to have a "high" functional impact on $H D A C 3$. VarioWatch assigns high functional impact to variants falling into an exonic splicing enhancer (ESE) site and into the protein domain. F-SNP database [24] showed that two out of four tools (ESRSearch and PESX) predicted an effect of rs2530223 on splicing regulation.

\section{Discussion}

Our exploratory study investigated for the first time the association between $H D A C 3$ variants, medication overuse, and other clinical variables in a sample of $23 \mathrm{MOH}$ patients. To this purpose, we sequenced exons, intron/ exon junctions, and 3'-5'UTR regions of the gene, and we identified six SNPs, all of which resulted cataloged in public databases. Our analyses showed a significant association of the $\mathrm{G}$ allele of rs2530223 with higher medication consumption, but not with attack frequency or intensity of headache. A recent study conducted in a small sample of $\mathrm{MOH}$ patients showed that the amount of acute medications taken for month was able to predict the prognosis at a 1-year follow-up [25]. This observation was confirmed by a recent prospective study conducted in a larger sample of $\mathrm{MOH}$ patients showing that overuse severity was associated with negative outcome at a 3-year follow-up [26]. Thus, understanding the basis of medication consumption in $\mathrm{MOH}$ patients appears to be of great relevance.

Our preliminary results suggest that rs2530223 could be implicated in excessive medication consumption independently of the necessity of acute medication for pain relief. The GG genotype of the same SNP has been associated with cigarette smoking in a Korean population of schizophrenia patients $[p=0.0054$; odds ratio (95\% confidence interval $)=4.33(1.41-13.25)$ ] [27]. Prevalence of smoking in schizophrenia patients is three times higher compared to general population, but carriers of the rs2530223 GG genotype seem particularly predisposed to develop this habit [27].

Although rs 2530223 is a synonym variant, it could be in strong linkage disequilibrium with rare functional variants that our study was not powered enough to detect. Moreover, VarioWatch predicted a high risk of functional impact of this SNP on HDAC3. Our results support previous evidence suggesting an involvement of genetic factors in predisposition to medication overuse in $\mathrm{MOH}$ patients [28].

Allele frequencies of rs2530223 are population specific, as the MAF in subjects with European, West African, or East Asian ancestry is 0.4, 0.16, and 0.63, respectively, accordingly to data from the 1000 Genome Project [29] obtained through VarioWatch [23]. Further studies in other ethnic groups are needed to assess the potential 
association of this SNP with medication consumption in other populations.

In our sample, subjects with the rs2530223 AA genotype were characterized by a lower headache frequency $(p=0.09)$. Thus, an alternative explanation for our results would be that subjects with this genotype show lower medication consumption due to a lower number of days/ month with headache. After correcting for headache frequency, the association between rs2530223 genotype and medication consumption was still significant $(p=0.03)$. While these results support our hypothesis that the SNP could play a role in medication overuse in these patients, the small sample size does not allow us to completely rule out a major contribution of headache frequency in driving significance. Findings from our study should be interpreted in light of the pilot nature of this investigation. The small sample size implemented could increase the risk for type 1 error and did not allow testing the effect of potentially relevant modifying factors in our model. Another limitation of the study is the small number of "AA" carriers in analysis under the recessive model. Strengths of our study include the prospective design and the choice to sequence $H D A C 3$ instead of target genotyping.

In conclusion, our pilot study suggests the involvement of $H D A C 3$ in excessive medication consumption in $\mathrm{MOH}$ patients. Our findings should be interpreted cautiously due to the limited sample size and might represent a first step of future extensive research aiming to clarify the role of $H D A C 3$ in medication overuse.

\section{Conclusions}

HDAC3 could be implicated in excessive medication consumption in $\mathrm{MOH}$ patients. These findings will serve as proof of concept for further investigations that will have to be performed on larger independent samples to confirm and extend our preliminary findings.

\section{Methods \\ Subjects}

This is an ancillary study from the sodium valproate in the treatment of medication overuse headache (SAMOHA) multicenter, randomized, double-blind, placebo-controlled study that enrolled $88 \mathrm{MOH}$ patients for a 3-month treatment period with VPA ( $800 \mathrm{mg} /$ day $)$ or placebo after a 6-day outpatient detoxification regimen, followed by a 3 -month follow-up. The 3-month responder rate (the proportion of patients achieving $\geq 50 \%$ reduction in the number of days with headache per month) was higher in the VPA (45.0 \%) than in the placebo arm (23.8\%) with an absolute difference of about $20 \%(p=0.043)$. VPA had safety and tolerability profiles comparable to placebo [22]. Informed written consent for the molecular study was obtained from 23 subjects included in the present work. All subjects undertook a venous blood draw before starting detoxification regimen and VPA therapy. The study was approved by the local Review Board. Table 1 shows the characteristics of the sample.

\section{Laboratory procedures}

Genomic DNA was extracted from peripheral blood leukocytes according to classical salting-out protocol. The human HDAC3 gene is located on chromosome $5 \mathrm{q} 31$ and comprises 15 exons, for a length of $16 \mathrm{~kb}$. The sequenced regions included all exons, intron/exon junctions, and $3^{\prime}-5$ 'UTR regions.

Sequencing was carried out using the Big Dye Terminator sequencing kit on an ABI-PRISM 3130XL Genetic Analyzer (Applied Biosystems) according to the manufacturer's protocol.

\section{Statistical analysis}

Sequences were analyzed with Sequence Scanner software v1.0 and aligned to the reference sequence of HDAC3 (UCSC Genome Bioinformatics) using BioEdit, version 7.2.5. Deviation from the HWE was tested with chi-square test. Polymorphisms with a MAF $<0.01$ were excluded from the analyses. Association between genotypes with continuous clinical characteristics and response to VPA was tested by multivariate analysis and Fisher's exact test, respectively. Sex, headache frequency, and response to VPA were tested in the model as covariates. All the analyses were carried out with Haploview version 4.2 [30] and SPSS version 20 (IBM Statistics). A $P$ value $<0.05$ was considered as statistically significant. Functional impact of associated variants was calculated with VarioWatch [23], a tool that assigns genomic variants a risk level on functional impact from "very low" to "very high". Analysis was conducted using standard parameters (selected region: $50 \mathrm{kbp}$ upstream and $50 \mathrm{kbp}$ downstream of SNP position). Effect of associated variants on splicing regulation was assessed with F-SNP [24], a database that predicts SNPs effect on splicing regulation by integrating information of four different tools: ESEfinder, RescueESE, ESRSearch, and PESX.

\section{Abbreviations \\ ESE: exonic splicing enhancer; HDAC3: histone deacetylases 3; HWE: Hardy- Weinberg equilibrium; MAF: minor allele frequency; $\mathrm{MOH}$ : medication overuse headache; SAMOHA: sodium valproate in the treatment of medication overuse headache; SNP: single-nucleotide polymorphisms; VPA: sodium valproate.}

\section{Competing interests}

No competing interests.

\section{Authors' contributions}

CP contributed in the study concept and design, analysis of data, and writing of the first draft of the manuscript. SC contributed in the acquisition, analysis, and interpretation of clinical data. DC performed the experiments and contributed in writing of the first draft of the manuscript. LMC contributed in selecting and collecting patients and biological samples from her Unit. AS contributed in data analysis and interpretation and revised the manuscript. GP contributed 
supervising the final version of the manuscript. IC contributed in selecting and collecting patients and biological samples from her Unit and in acquisition, analysis, and interpretation of clinical data. PC contributed in coordinating the clinical and molecular studies. MDZ coordinated the molecular study and contributed to study design and revising of the manuscript. PS coordinated the clinical study and contributed to study design and revising of the manuscript. All authors read and approved the final manuscript.

\section{Financial support}

This research was partly funded by "Agenzia Italiana del Farmaco" (AIFA) in the context of the project "AREA: Pharmacoepidemiological studies aimed at defining the benefit-risk profile of treatments and the impact of strategies for improving the appropriateness of drug use; TOPIC: Studies on pharmacological treatments of chronic headache" (announcement year 2006).

\section{Author details}

${ }^{1}$ Section of Neurosciences and Clinical Pharmacology, Department of Biomedical Sciences, University of Cagliari, SP8 Km. 0,700, 09042 Monserrato (CA), Italy. ${ }^{2}$ Neurologic Clinic, University of Perugia, Perugia, Italy. ${ }^{3}$ Headache and Cerebrovascular Diseases Center, Ospedale S. Eugenio, Rome, Italy. ${ }^{4}$ Department of Pharmacy, University of Patras, School of Health Sciences, Patras, Greece. ${ }^{5}$ IRCCS Fondazione "S. Lucia", Rome, Italy. ${ }^{6}$ Institute of Neuroscience, CNR, Cagliari, Italy.

Received: 20 July 2015 Accepted: 26 October 2015

Published online: 05 November 2015

\section{References}

1. Terrazzino S, Tassorelli C, Sances G, Allena M, Viana M, Monaco F, et al. Association of haplotype combination of serotonin transporter gene polymorphisms with monthly headache days in $\mathrm{MOH}$ patients. Eur J Neurol. 2012;19:69-75.

2. Russell MB, Lundqvist C. Prevention and management of medication overuse headache. Curr Opin Neurol. 2012;25:290-5.

3. Katsarava Z, Fritsche G, Muessig M, Diener HC, Limmroth V. Clinical features of withdrawal headache following overuse of triptans and other headache drugs. Neurology. 2001;57:1694-8.

4. Cargnin S, Pautasso C, Viana M, Sances G, Mittino D, Cantello R, et al. Association of RAMP1 rs7590387 with the risk of migraine transformation into medication overuse headache. Headache. 2015;55:658-68.

5. Cargnin S, Viana M, Sances G, Bianchi M, Ghiotto N, Tassorelli C, et al. Combined effect of common gene variants on response to drug withdrawal therapy in medication overuse headache. Eur J Clin Pharmacol. 2014;70:1195-202.

6. Cargnin S, Viana M, Ghiotto N, Bianchi M, Sances G, Tassorelli C, et al. Functional polymorphisms in COMT and SLC6A4 genes influence the prognosis of patients with medication overuse headache after withdrawal therapy. Eur J Neurol. 2014;21:989-95.

7. Bigal ME, Serrano D, Buse D, Scher A, Stewart WF, Lipton RB. Acute migraine medications and evolution from episodic to chronic migraine: a longitudinal population-based study. Headache. 2008:48:1157-68

8. Tassorelli C, Jensen R, Allena M, De Icco R, Sances G, Katsarava Z, et al. A consensus protocol for the management of medication-overuse headache: evaluation in a multicentric, multinational study. Cephalalgia. 2014;34:645-55.

9. Biagianti B, Grazzi L, Gambini O, Usai S, Muffatti R, Scarone S, et al. Decisionmaking deficit in chronic migraine patients with medication overuse. Neurol Sci. 2012;33:S151-5.

10. Fumal A, Laureys S, Di Clemente L, Boly M, Bohotin V, Vandenheede M, et al. Orbitofrontal cortex involvement in chronic analgesic-overuse headache evolving from episodic migraine. Brain. 2006;129:543-50.

11. London ED, Ernst M, Grant S, Bonson K, Weinstein A. Orbitofrontal cortex and human drug abuse: functional imaging. Cereb Cortex. 2000;10:334-42.

12. Ferraro S, Grazzi L, Muffatti R, Nava S, Ghielmetti F, Bertolino N, et al. In medication-overuse headache, fMRI shows long-lasting dysfunction in midbrain areas. Headache. 2012;52:1520-34.

13. Calabresi P, Cupini LM. Medication-overuse headache: similarities with drug addiction. Trends Pharmacol Sci. 2005;26:62-8.

14. Fuh $J \mathrm{~L}$, Wang SJ. Dependent behavior in patients with medication-overuse headache. Curr Pain Headache Rep. 2012;16:73-9.
15. de Ruijter AJ, van Gennip AH, Caron HN, Kemp S, van Kuilenburg AB. Histone deacetylases (HDACs): characterization of the classical HDAC family. Biochem J. 2003;370:737-49.

16. McQuown SC, Barrett RM, Matheos DP, Post RJ, Rogge GA, Alenghat T, et al. HDAC3 is a critical negative regulator of long-term memory formation. J Neurosci. 2011;31:764-74.

17. Robbins TW, Ersche KD, Everitt BJ. Drug addiction and the memory systems of the brain. Ann N Y Acad Sci. 2008;1141:1-21.

18. Malvaez M, McQuown SC, Rogge GA, Astarabadi M, Jacques V, Carreiro S, et al. HDAC3-selective inhibitor enhances extinction of cocaine-seeking behavior in a persistent manner. Proc Natl Acad Sci U S A. 2013;110:2647-52.

19. Li X, Rubio FJ, Zeric T, Bossert JM, Kambhampati S, Cates HM, et al. Incubation of methamphetamine craving is associated with selective increases in expression of BDNF and trkb, glutamate receptors, and epigenetic enzymes in cue-activated fos-expressing dorsal striatal neurons. J Neurosci. 2015;35:8232-44.

20. Yurekli VA, Akhan G, Kutluhan S, Uzar E, Koyuncuoglu HR, Gultekin F. The effect of sodium valproate on chronic daily headache and its subgroups. J Headache Pain. 2008;9:37-41.

21. Blumenfeld AM, Schim JD, Chippendale TJ. Botulinum toxin type A and divalproex sodium for prophylactic treatment of episodic or chronic migraine. Headache. 2008;48:210-20.

22. Sarchielli P, Messina P, Cupini LM, Tedeschi G, Di Piero V, Livrea P, et al. Sodium valproate in migraine without aura and medication overuse headache: a randomized controlled trial. Eur Neuropsychopharmacol. 2014;24:1289-97.

23. Cheng YC, Hsiao FC, Yeh EC, Lin WJ, Tang CY, Tseng HC, et al. VarioWatch: providing large-scale and comprehensive annotations on human genomic variants in the next generation sequencing era. Nucleic Acids Res. 2012;40:W76-81.

24. Lee PH, Shatkay H. F-SNP: computationally predicted functional SNPs for disease association studies. Nucleic Acids Res. 2008;36:D820-4.

25. Radat F, Chanraud S, Di Scala G, Dousset V, Allard M. Psychological and neuropsychological correlates of dependence-related behaviour in medication overuse headaches: a one year follow-up study. J Headache Pain. 2013;14:59.

26. Sances G, Galli F, Ghiotto N, Allena M, Guaschino E, Frustaci A, et al. Factors associated with a negative outcome of medication-overuse headache: a 3year follow-up (the 'CARE' protocol). Cephalalgia. 2013;33:431-43.

27. Kim T, Park JK, Kim HJ, Chung JH, Kim JW. Association of histone deacetylase genes with schizophrenia in Korean population. Psychiatry Res. 2010;178:266-9.

28. Di Lorenzo C, Di Lorenzo G, Sances G, Ghiotto N, Guaschino E, Grieco GS, et al. Drug consumption in medication overuse headache is influenced by brain-derived neurotrophic factor Val66Met polymorphism. J Headache Pain. 2009;10:349-55.

29. 1000 Genomes Project Consortium. A map of human genome variation from population-scale sequencing. Nature. 2010;467:1061-73.

30. Barrett JC, Fry B, Maller J, Daly MJ. Haploview: analysis and visualization of LD and haplotype maps. Bioinformatics. 2005;21:263-5.

31. Rambaldi D, Ciccarelli FD. FancyGene: dynamic visualization of gene structures and protein domain architectures on genomic loci. Bioinformatics. 2009;25:2281-2.

\section{Submit your next manuscript to BioMed Central and take full advantage of:}

- Convenient online submission

- Thorough peer review

- No space constraints or color figure charges

- Immediate publication on acceptance

- Inclusion in PubMed, CAS, Scopus and Google Scholar

- Research which is freely available for redistribution 